\title{
Characterization and sequence analysis of the $l s g$ (LOS synthesis genes) locus from Haemophilus influenzae type b
}

\author{
R. McLaughlin, N.-G. Lee, Y. Abu Kwaik, S. M. Spinola, M. A. Apicella \\ Department of Microbiology, The University of Oklahoma Health Sciences Center, OK, USA, Department of \\ Microbiology, University of lowa, Iowa City, IA, USA, Department of Microbiology and Immunology, University of \\ Michigan, Ann Arbor, MI, USA and Department of Medicine, Division of Infectious Diseases, University of Indiana, \\ Indianapolis, IN, USA
}

SUMMARY. Analysis of the lsg (LOS synthesis genes) cluster in Escherichia coli strain $\mathrm{K} 12$ and mutations in the $\mathbf{l s g}$ locus in Haemophilus influenzae type $b$ indicated the presence of 3 regions responsible for sequential modifications of $E$. coli lipopolysaccharide (LPS). Sequencing of the $l s g$ region yielded 7,435 bp that encompassed 7 complete and 1 partial open reading frames (ORFs 1-8). The predicted product of ORF1 had homology to the consensus sequence of cytochrome b proteins ( $21 \%$ identity, $51 \%$ similarity) and to other transmembrane proteins. The products of ORF5 and ORF6 share overall $23 \%$ identity and $49 \%$ similarity with each other. The ORF6 protein had high homology with the product of ORF275 of the $E$. coli $\mathrm{rfb}$ gene cluster (40\% identity, $\mathbf{5 8 \%}$ similarity), whose function is not known. Multiple sequence alignment of the ORF5 and ORF6 proteins with the RfbB, RfbJ and $R f b X$ proteins revealed conserved motifs over the $\mathrm{N}$-terminal half region of all these proteins. The products of ORF7 and ORF8 are homologous with Azotobacter vinelandii MolA protein ( $30 \%$ identity, $51 \%$ similarity) and MolB protein (26\% identity, $48 \%$ similarity), respectively. The promoter regions of ORF1, 7 and 8 were determined by primer extension analysis and found to be similar to bacterial $\sigma^{70}$-dependent promoters. ORF7 and ORF8 are transcribed into diverse orientation. At least 5 of the encoded proteins have been identified using coupled $E$. coli transcription/translation system and labeling with [ ${ }^{35} \mathrm{~S}$ ]-methionine. We conclude that the genetic organization of the $l s g$ biosynthesis pathway involves multiple operons that lead to the assembly of an $H$. influenzae LOS structure.

The lipooligosaccharides (LOSs) of Haemophilus influenzae type b (Hib) are important virulence factors for this human pathogen. ${ }^{1-3}$ The oligosaccharide region of Hib LOS is composed of a complex array of stable epitopes and epitopes that phase vary at frequencies from

R. McLaughlin PhD, Department of Microbiology, The University of Oklahoma Health Sciences Center, OK 73190, USA, N.-G. Lee PhD. M. A. Apicella MD, Department of Microbiology, University of Iowa. Iowa City, IA 52242, USA, Y. Abu Kwaik PhD, Department of Microbiology and Immunology, University of Michigan, Ann Arbor, MI 48109, USA, S. M. Spinola MD, Department of Medicine, Division of Infectious Diseases, University of Indiana, Indianapolis, IN 46202, USA.

Correspondence to Michael A. Apicella MD, Department of Microbiology, University of Iowa, Bowen Science Building Rm 3-401, 51 Newton Road, lowa City, Iowa 52242, USA.
$10^{-2}$ to $10^{-3} \cdot 1,2,4$ Elucidation of the regulatory and biosynthetic genes responsible for LOS oligosaccharide biosynthesis and phase variation has recently begun. ${ }^{5-9}$

Our laboratory has described a cloned Hib gene cluster ( $l s g$ : LOS synthesis genes) that is responsible for assembly of a series of saccharide components on the Escherichia coli K-12 lipopolysaccharide (LPS). ${ }^{4,5,7,10}$ The $l s g$ locus is contained within a $7.4 \mathrm{~kb} \mathrm{BamHI-PstI}$ fragment isolated from Hib strain A2. Three overlapping subclones of the $l s g$ locus produce sequential LPS modifications in $E$. coli K-12. ${ }^{5}$ Initial modification of the $E$. coli LPS requires the presence of the $2.8 \mathrm{~kb} P s t \mathrm{I}-$ $S p h \mathrm{I}$ fragment that assembles a $0.4 \mathrm{kDa}$ oligosaccharide on a $4.1 \mathrm{kDa}$ LPS structure. The Pst $\mathrm{I} S p h \mathrm{I}$ fragment combined with the adjacent $2.7 \mathrm{~kb} S p h \mathrm{~L}-$ HindIII fragment leads to assembly of an additional $0.6 \mathrm{kDa}$ oligo- 
saccharide on the modified structure. A $1.4 \mathrm{kDa}$ modification to $E$. coli LPS is generated by addition of the $1.7 \mathrm{~kb}$ HindIII-BamHI fragment to the PstI-HindIII fragment. Generation of isogenic Hib mutants of the lsg locus has verified its involvement in LOS biosynthesis. ${ }^{10}$ The chemical structure of $H$. influenzae LOS is now being elucidated. ${ }^{11}$ The deep core region contains a branched tri-heptose structure. It appears that each heptose can be substituted by an oligosaccharide chain. This complexity has made analysis of the terminal LOS sugars difficult and has hindered assignment of specificities to the biosynthesis genes involved in their production.

In this study, we present the DNA sequence analysis and further characterization of the $l s g$ locus. The sequence contained 7 complete and 1 partial open reading frames (ORFs), some of which encode proteins homologous to enterobacterial $\mathrm{Rfb}$ proteins. The promoters of ORF1, ORF7 and ORF8 were localized by promoter analysis and found to be similar to bacterial $\sigma^{70}$ - dependent promoters. In vitro translation studies confirmed at least 5 of the gene products predicted by the ORFs.

\section{MATERIALS AND METHODS}

\section{Bacterial strains and growth conditions}

$H$. influenzae type b (Hib) strain A2 was grown on chocolate agar supplemented with isovitalex or brain heart infusion agar supplemented with $4 \%$ Fildes reagent (sBHI) (Difco Laboratories) at $35^{\circ} \mathrm{C}$ in a $5 \% \mathrm{CO}_{2}$ atmosphere or in sBHI broth at $37^{\circ} \mathrm{C}$ with agitation. Chloramphenicol $(2 \mu \mathrm{g} / \mathrm{ml})$ or kanamycin $(20 \mu \mathrm{g} / \mathrm{ml})$ was added to $\mathrm{sBHI}$ medium for selection and maintenance of Hib isolates containing the mini- $\mathrm{Tn} 3(\mathrm{Cm})$ transposon or kanamycin resistance $\left(\operatorname{kan}^{\mathbf{r}}\right)$ cassette, respectively. $E$. coli strains were routinely cultured at $37^{\circ} \mathrm{C}$ using LB agar or broth with appropriate antibiotics.

\section{Monoclonal antibodies}

Monoclonal antibody (MAb) 6E4 has been previously described. $^{7}$ MAb $2 F 2$ is an IgG murine monoclonal antibody generated against the LOS from $H$. influenzae non-typable strain 2019. Both MAbs were detected after binding with protein A conjugated to horseradish peroxidase (Zymed Laboratories). Monoclonal antibody binding in ELISA assay was classified as normal (> 75\%), reduced (between $25-75 \%$ ) or negative $(<10 \%)$ as compared with the parental strain. All values are the average of at least 10 replicate samples from a single assay. Each assay was performed at least twice.

\section{DNA manipulation}

Plasmid and chromosomal DNA isolation and Southern hybridization mapping were performed as previously described. $^{5}$ T4 DNA ligase and restriction enzymes were used according to the manufacturer's suggestions (GIBCO BRL and Promega). Haemophilus cells were made competent and transformed according to the $\mathrm{M}$ IV medium procedure. ${ }^{12}$ Transformation of $E$. coli strains with plasmid DNA was carried out by the $\mathrm{CaCl}_{2}$ method. ${ }^{13}$

\section{Mutagenesis}

Transposon mutagenesis of the lsg locus was accomplished using shuttle mutagenesis as previously described. ${ }^{5,14}$ The location of transposon insertion sites was determined by genomic Southern hybridization.

\section{Phenotypic analysis}

The immunochemical phenotypes of $l s g$ mutants were determined by ELISA and Western blot as previously described. ${ }^{10}$ The LOS banding patterns of the mutant strains were determined by digestion of whole cells with proteinase $\mathrm{K},{ }^{15}$ separation by sodium dodecyl sulfate-polyacrylamide gel electrophoresis (SDS-PAGE) using tricine as the trailing ion ${ }^{16}$ and detection by silver staining. ${ }^{17}$

\section{DNA sequencing and sequence analysis}

Double-stranded DNA was sequenced by the dideoxynucleotide termination method ${ }^{18}$ labeled with $\left[\alpha-{ }^{35} S\right]$ dATP using the Sequenase II kit (United States Biochemicals). Sequence of the entire $7.4 \mathrm{~kb}$ region of the $l s g$ locus was accomplished by generating plasmids containing specific restriction fragments and by exonuclease III digestion of various subclones. ${ }^{5}$ Specific oligonucleotides were also used to sequence several regions.

The complete $7.4 \mathrm{~kb}$ sequence was assembled using the Fragment Assembly System contained in the Genetics Computer Group (GCG) sequence analysis software package. ${ }^{19}$ Analysis of the $l s g$ sequence was performed using various programs of the GCG package. Homology search with the deduced amino acid sequences within the SWISS-PROT and Genpept databases was done with GENBANK Online Service using the FASTA algorithm. ${ }^{20}$ Protein sequence alignment was done using the BESTFIT program of the GCG package.

\section{Primer extension analysis}

RNA was extracted from Hib A2 grown in sBHI broth to $\mathrm{OD}_{600} 0.6$ by sonication, digestion with proteinase $\mathrm{K}$ and phenol/chloroform extraction followed by ethanol precipitation as described. $^{13}$ The purified RNA was quantitated spectrophotometrically and the quality of RNA was confirmed on a formaldehyde-agarose gel stained with ethidium bromide.

Primer extension analysis was carried out using the 
Primer Extension Kit (Promega) following the manufacturer's instructions except for annealing, which was done by heating the reaction mixture to $70^{\circ} \mathrm{C}$, incubating at $60^{\circ} \mathrm{C}$ for $20 \mathrm{~min}$ and then slowly cooling down to room temperature. $40 \mu \mathrm{g}$ of RNA was used for each reaction and the reaction products were precipitated in ethanol, dissolved in loading dye, and loaded on a $6 \%$ sequencing gel. The dideoxy-sequencing ladder made with the primer was used as a marker to confirm the position of the primer extended products.

\section{In vitro transcription/translation analysis}

Plasmids containing the entire $l s g$ locus or various restriction fragments were purified by cesium chloride buoyant density gradient ${ }^{13}$ and analyzed in a coupled transcription/translation system (Promega) using translation grade [ $\left.{ }^{35} \mathrm{~S}\right]$-methionine (Amersham). Translation products were separated on a $20 \%$ acrylamide gel by SDS-PAGE. ${ }^{21}$ Low molecular weight $\left[{ }^{14} \mathrm{C}\right]$-labeled Rainbow standards (Amersham) were used to generate a linear regression curve between $14.3-46 \mathrm{kDa}$ for determination of relative molecular weights.

\section{Nucleotide sequence accession number}

The DNA sequence described has been deposited at GenBank with the accession number M94855.

\section{RESULTS}

\section{Nucleotide sequence of the $l s g$ locus}

The 7.4 kb lsg locus was completely sequenced from both strands. The fragment had a total length of 7435 bp that encompassed 7 complete and 1 partial open reading frames (ORFs1-8) determined with the FRAMES program. ${ }^{19}$ All ORFs were transcribed from left to right with the exception of ORF7 (Fig. 1A). All ORF coding regions had the high AT content characteristic of the Hib genome.

The sequence data suggested that ORFs $1-3$ and 4-6 comprised 2 operons. The predicted ATG start of ORF2 overlapped the TAA stop codon of ORF1 by 2 bases. Likewise, ORF2 and ORF3 were separated by only 1 base. ORF1 and ORF3 were translated in the same reading frame, while ORF2 was translated in an alternate frame. Possible ribosomal binding sites (RBS), based on homology with known RBS and the Shine-Dalgarno consensus sequence, ${ }^{22}$ were identified upstream of the predicted start of the 3 ORFs.

ORFs 4-6 were separated from the first operon by an intercistronic region of 359 bases. 11 bases separated ORF4 and ORF5 and only 1 base separated ORF5 from ORF6. A RBS was not identified upstream of the first start codon for ORF4. However, a potential RBS was located upstream of a second ATG codon $33 \mathrm{bp}$ from the first start. This suggested that the second ATG may be the true start codon for ORF4. ORF5 and
ORF6 each had a possible RBS located upstream of their predicted start codons, although the ORF6 RBS consensus was weak. ORFs 4-6 were translated in different reading frames.

ORF7 and ORF8 were divergently transcribed from a 137 bp intercistronic region. Potential ribosomal binding sites were identified 7 bases upstream of each ORF.

\section{Identification of sequences having homology with the $l s g$ ORFs}

Search for homologous sequences with the deduced amino acid sequences of the lsg ORFs within the SWISS-PROT and Genpept databases was done with GENBANK Online Service using the FASTA algorithm. ${ }^{20}$ Homologies were found with the predicted products of ORFs $1,5,6,7$ and 8 but no significant homologies to any sequences were obtained to the products of ORFs 2,3 or 4 .

The sequence having the highest homology with the ORF1 protein was a 403 amino acid (AA) consensus sequence compiled from 37 complete sequences of cytochrome $b$ present in the SWISS-PROT database. The consensus closely matched the predicted length of the ORFI protein (402 AA) and shared $21 \%$ identity and $51 \%$ similarity. The homology was not restricted to any particular region but evenly distributed throughout the protein sequences. A hydrophobicity profile of the ORF1 protein using Kyte-Doolittle algorithm ${ }^{23}$ showed a striking similarity to that of the cytochrome b consensus sequence (Fig. 2). Other homologous proteins included the NADH-dehydrogenase (ubiquinone) chain 4 ( $24 \%$ identity, $53 \%$ similarity, ), the formate hydrogenase subunit $\mathrm{HycD}$ ( $16 \%$ identity, $51 \%$ similarity) and the photosynthesis II D2 protein (17\% identity, $46 \%$ similarity). All homologous sequences were transmembrane proteins, the majority of which may act as or in conjunction with electron carriers.

The ORF5 protein had $22 \%$ identity and $46 \%$ similarity to the undefined ORF14.1 protein of the Salmonella typhi Ty2 $r b$ gene cluster and the equivalent ORF from $S$. typhimurium LT2. The ORF14.1 protein has been identified by mutational analysis as involved in assembly of the species specific $O$ antigen oligosaccharide. ${ }^{24}$ The ORF6 protein had high homology with the product of ORF275 of the $E$. coli $r b$ gene cluster ( $40 \%$ identity, $58 \%$ similarity), whose function is not known yet. $^{25}$ The ORF5 and ORF6 proteins shared homology with each other ( $23 \%$ identity, $49 \%$ similarity). Homology search revealed that the ORF5 and ORF6 proteins were homologous with the $R f b B, R f b J$ and $\mathrm{RfbX}$ proteins encoded in the $r b$ gene cluster, but the overall homology was rather low (18-24\% identity, 41$48 \%$ similarity). Multiple sequence alignment, however, revealed several motifs conserved in the $\mathrm{N}$-terminal half of these proteins (Fig. 3).

The products of ORF7 and ORF8 shared homology with the MolA protein ( $30 \%$ identity, $51 \%$ similarity) and the MolB protein ( $26 \%$ identity, $48 \%$ similarity), 
respectively, encoded in the gene cluster which is involved in molybdenum transport in Azotobacter vinelandii (Fig. 4). ${ }^{26}$

\section{Localization of the promoter regions of the $l s g$ ORFs}

In order to determine the promoter regions for the $l s g$ ORFs, primer extension analysis was carried out using oligonucleotides complementary to the regions of DNA just downstream of the putative translation initiation codon ATGs (whose sequences are shown in Figure 6). The transcription start point of ORF1 was mapped to 54 to 56 and 51 nucleotides (nt) upstream of the putative ATG codon (Fig. 5A). It is not clear whether the tran- scription initiates at 2 closely spaced sites or whether the downstream site is an artifact caused by early fall off of reverse transcriptase. The promoter region contained a sequence (TAAAAT) homologous to the consensus -10 element conserved among the bacterial $\sigma^{70}$-dependent promoters (Fig. 6). The further upstream region also contained a sequence which agrees with 4 of $6 \mathrm{nt}$ for the consensus -35 element, but the distance to the -10 element was only $13 \mathrm{nt}$. Primer extension with the primer for ORF7 yielded a major band at $38 \mathrm{nt}$ upstream of the putative ATG codon and several minor bands. The promoter region contained the -10 element with the distance of $8 \mathrm{nt}$ to the transcription start site, but no -35 like sequence was found (Figs 5B \& 6). For
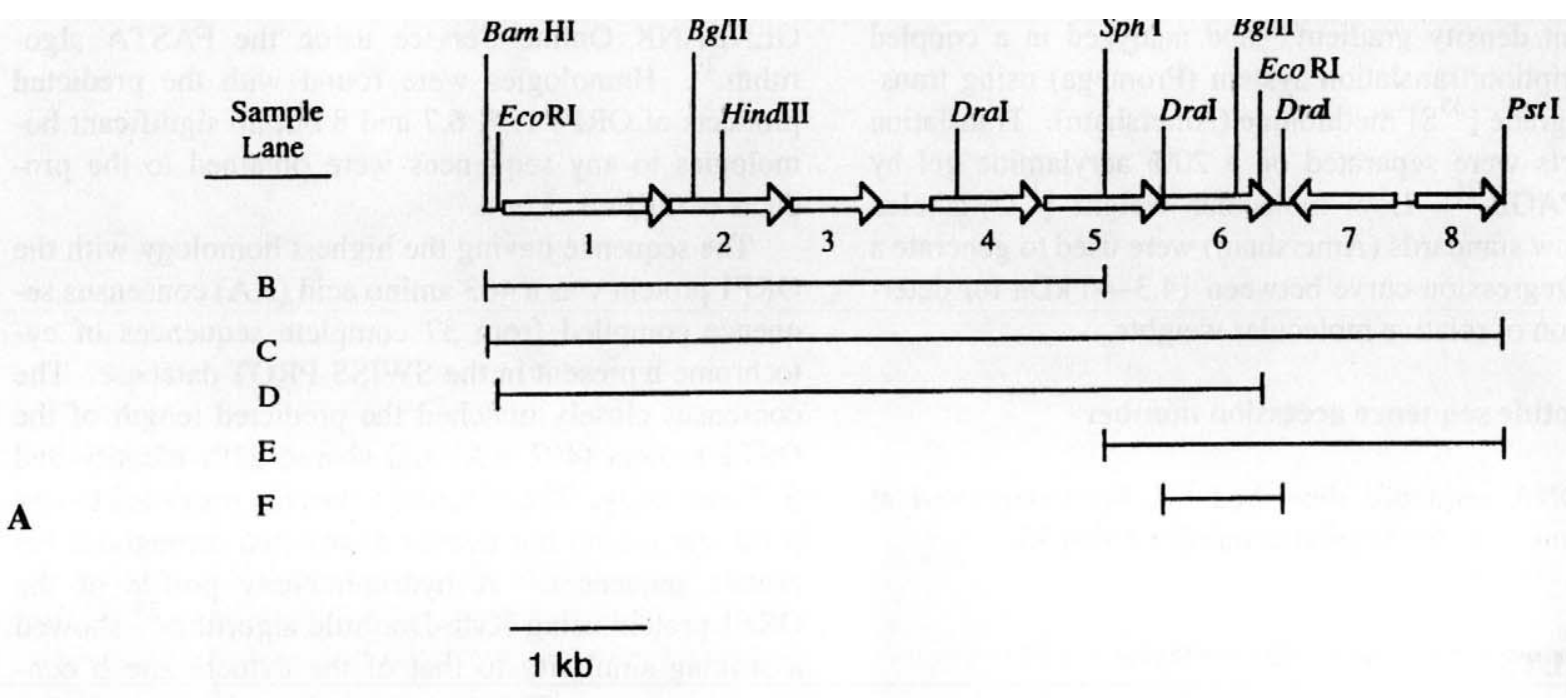

A

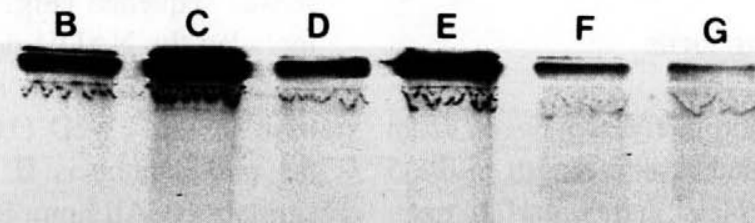

30

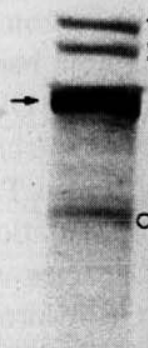

21

B

14
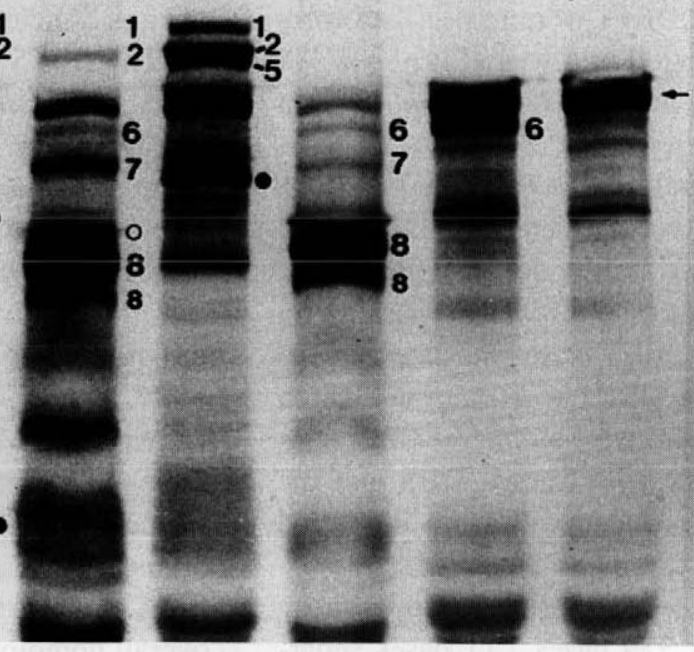

Fig. 1 - (A) Restriction map of the lsg locus showing deduced open reading frames (ORFs). Plasmids containing the restriction fragments indicated were used for in vitro transcription/translation assay. Numbers depicted below the map correspond to the respective ORFs. (B) In vitro transcription/translation of the $l s g$ locus. Lane A, low molecular weight protein standards; lanes B-F, translation products of $l s g$ subclones corresponding to those depicted in 1A; lane G, pGEM-3zf+ (Promega). Numbers appearing next to protein bands correspond to translation products of ORFs 1-8, respectively. The open circles denote weak bands that may correspond to ORF 3 . The solid circles indicate truncated peptides, or peptides fused with lacZ $\alpha$ peptide. The band indicated by the arrow represents the $\beta$-lactamase precursor and possibly the ORF 4 translation product. 


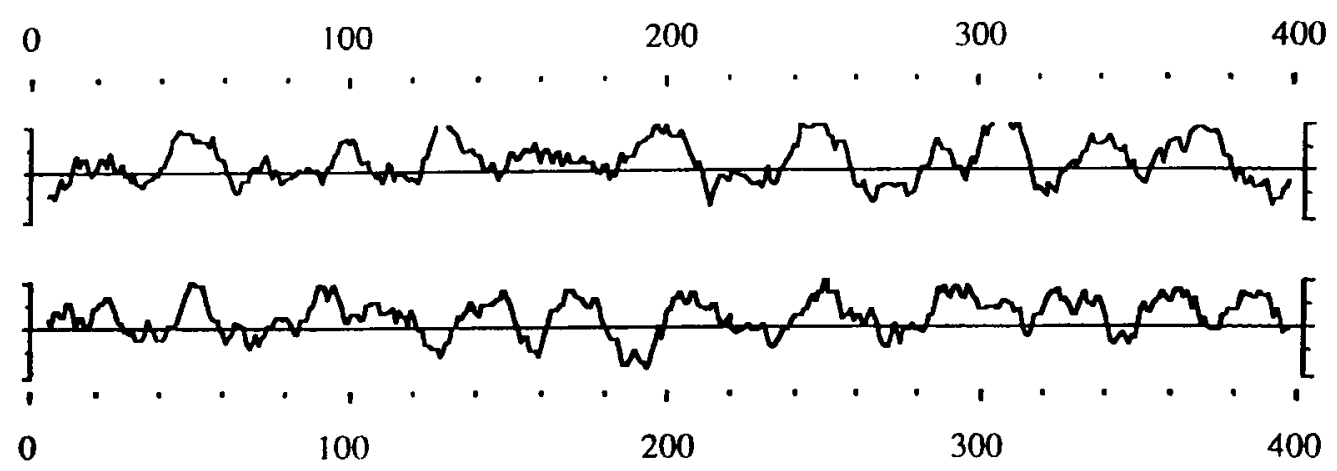

Fig. 2 - Hydrophobicity plot of the cytochrome b consensus sequence and the ORF 1 protein derived with the Pepplot program using the KyteDoolittle algorithm. The upper graph is the plot of the cytochrome $b$ consensus, the lower graph is that of ORF1 protein. Values above the $x$-axis indicate hydrophobic regions of the proteins.

ORF8 2 major transcription initiation sites were found at 28 and $20 \mathrm{nt}$ upstream of the putative ATG codon and the upstream sequence contained a perfect -10 element. Further upstream of this putative -10 element are 2 possible -35 sequences, 12 and $23 \mathrm{nt}$ from the -10 region, which agree with 4 of $6 \mathrm{nt}$ of the consensus -35 motif (Figs 5C \& 6). The primer extension reactions for all 3 ORFs with RNA extracted from $E$. coli transformed with a plasmid containing the whole $7.4 \mathrm{~kb} / \mathrm{sg}$ locus yielded the same band pattern as those with Hib A2 RNA, though the transcription levels were lower in E. coli (Fig. 5).

\section{Translational analysis of the $l s g$ locus}

A coupled transcription/translation system was used to confirm the production of proteins from the deduced ORFs of the lsg locus. Various plasmids containing fragments of the $l s g$ locus were constructed (Fig. 1A) and used for in vitro translation. Labeled proteins having $\mathrm{M}_{\mathrm{r}}$ values approximating that of the deduced proteins for ORFs 2 and 5 through 8 were identified (Fig. 1B, Table). A weak band approximating the $M_{r}$ of ORF3 was also observed. A protein equivalent to ORF4 was not identified due to probable co-migration with the $\beta$-lactamase precursor protein.

The deduced protein for ORF1 had a molecular weight of approximately $46 \mathrm{kDa}$, but the protein resulting from translation of ORF1 had a $\mathbf{M}_{\mathbf{r}}$ of approximately $40 \mathrm{kDa}$. Examination of the primary sequence failed to reveal possible internal start codons, and the secondary structural prediction for ORF1 gave no indication of a leader sequence. ORF8 encoded a peptide of approximately $22 \mathrm{kDa}$. The secondary structure prediction for ORF8 indicated a highly hydrophobic $\mathrm{NH}_{2}-$ terminal region that contained 35 amino acids and was

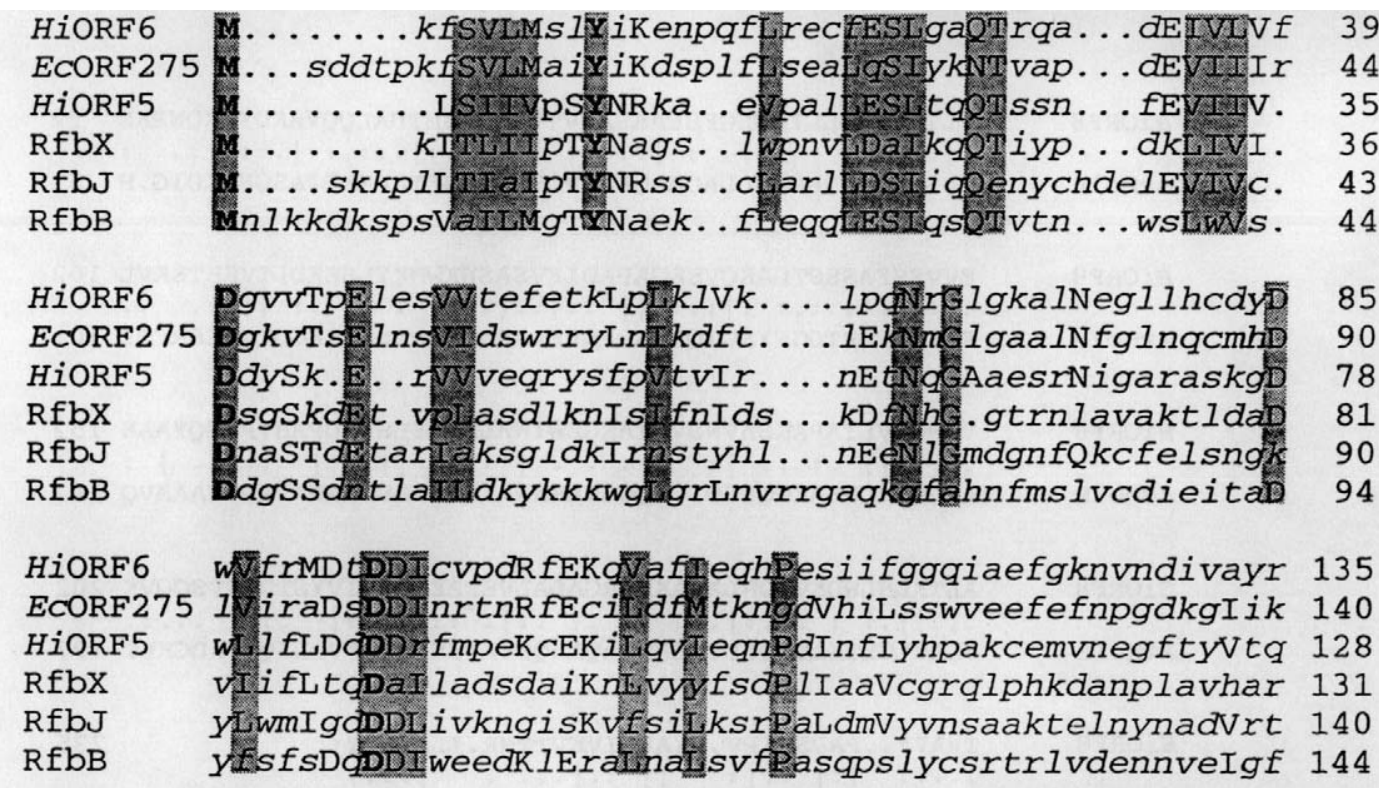

Fig. 3 - Multiple sequence alignment of proteins homologous to the products of the ORF 5 and 6 . Gaps inserted for optimal alignments are indicated by dots. The C-terminal sequences have been omitted because of lack of sequence similarity among HiORFs and Rfb proteins. Residues conserved in all 6 proteins are in bold, residues identical or similar (I-L-M-V, D-E, K-R, N-Q, S-T, F-Y) in more than 5 proteins are shaded and residues identical or similar in more than 4 proteins are capitalized. HiORF6, Hib A2 strain lsg locus ORF6 protein; EcORF275, the product of ORF275 of an unknown function present in E. coli $r f b$ region (L04596); HiORF5, Hib A2 strain lsg locus ORF5 protein; RfbX, S. typhimurium RfbX protein (P26403); RfbJ, S. choleraesuis RfbJ protein (S22615); RfbB, Y. enterocolitica RfbB protein (S28578). The codes in the parentheses are the accession numbers for DNA or protein sequence libraries. 
characteristic of a signal peptide. Analysis of the translation products from clones containing the ORF8 region revealed 2 bands with $M_{r}$ values of 23 and $22 \mathrm{kDa}$. Cleavage of the proposed signal peptide of ORF8 would result in a protein of approximately $21 \mathrm{kDa}$.

\section{Mutagenesis of the EcoRI-Pst I fragment}

We have previously reported the generation and analysis of transposon insertion mutants within the internal EcoRI fragment of the $l s g$ locus. ${ }^{5,10}$ These insertions led to alterations to the Hib LOS defined by reduced or negative binding of $\mathrm{MAb} 6 \mathrm{E} 4$. We now report the analysis of additional transposon insertion mutants within the terminal $1.7 \mathrm{~kb}$ EcoRI-Pst fragment. In addition, we have generated several $\operatorname{kan}^{\mathrm{r}}$ cassette insertions and replacement/insertions within the $l s g$ locus.

Transposon insertions within the $1.7 \mathrm{~kb}$ EcoRI-Pst fragment of the $l s g$ locus resulted in alterations to LOS epitopes that were detected with MAbs 6E4 and 2F2. Transposon insertions that were localized to the $3^{\prime}$ end of ORF6 reduced the MAb $6 E 4$ and $2 F 2$ binding to LOS from these mutants more than $25 \%$ compared to that of the parental strain (Fig. 7A). These values were

$\mathbf{A}$

\begin{tabular}{|c|c|}
\hline AvMolA & 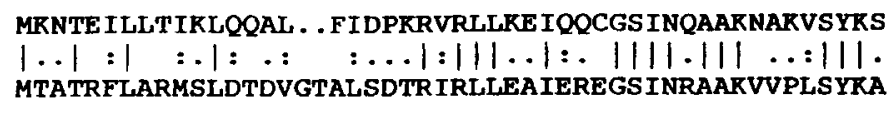 \\
\hline BIORF 7 & 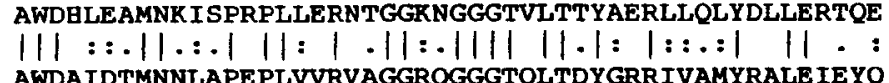 \\
\hline $\begin{array}{l}\text { BIORF7 } \\
\text { AvMOLA }\end{array}$ & 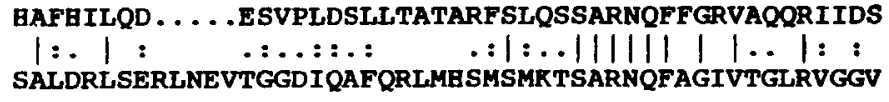 \\
\hline BIORF7 & 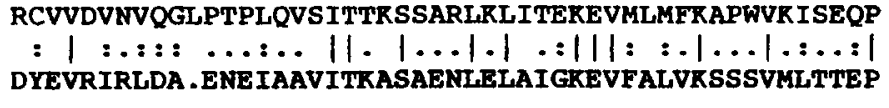 \\
\hline BIORF7 & 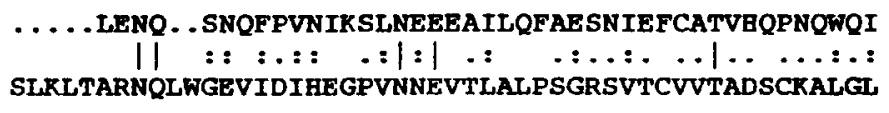 \\
\hline HIORF 7 & $\begin{array}{l}\text { GQQVW. . IHIDQEQIILATLG* } \\
: \ldots|\quad: \quad: \quad=||| \ldots \mid \\
\text { APGVAACAFFKSSVILAVYG* }\end{array}$ \\
\hline
\end{tabular}

$\mathbf{B}$

\begin{tabular}{|c|c|}
\hline BIORF 8 & 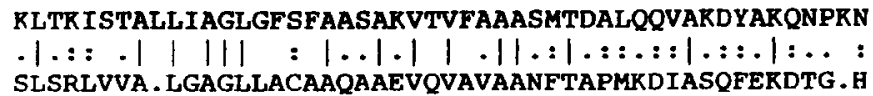 \\
\hline BiORF 8 & 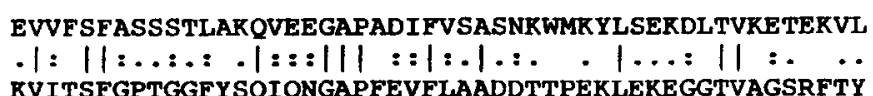 \\
\hline GiORF 8 & 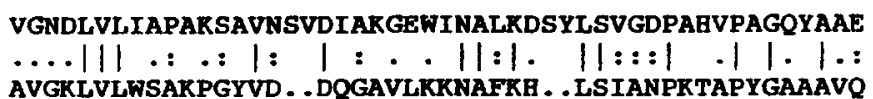 \\
\hline BIORF & 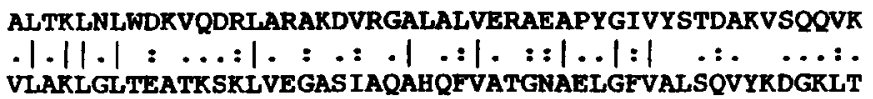 \\
\hline GIORF 8 & 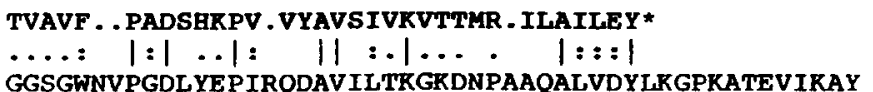 \\
\hline
\end{tabular}

Fig. 4 - Comparison of the predicted amino acid sequences of the ORF7 product (HiORF7) and the A. vinelandii MolA protein (AvMolA) (A) and the ORF8 product (HiORF8) and the A. vinelandii MolB protein (AvMolB) (B). The DNA sequence of ORF8 past PstI site was completed by single stranded sequencing. Identical residues are denoted by bars and similar residues by dots. Gaps were inserted for maximal alignments. Asterisks indicate stop codons. 

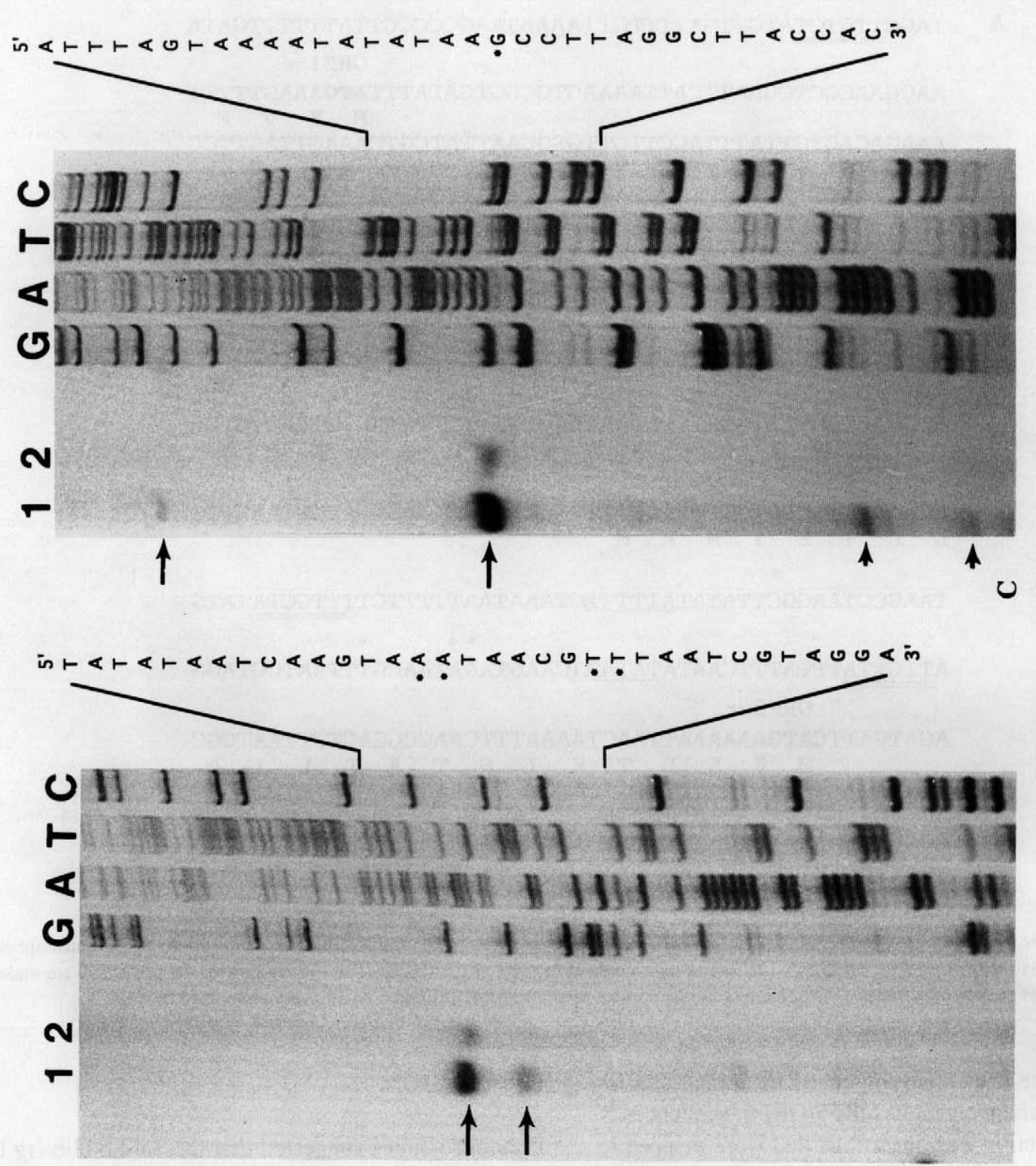

密

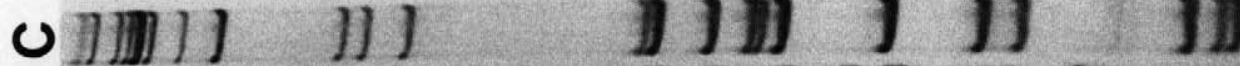

范 造

空苞吉

x 总

总焉营

总

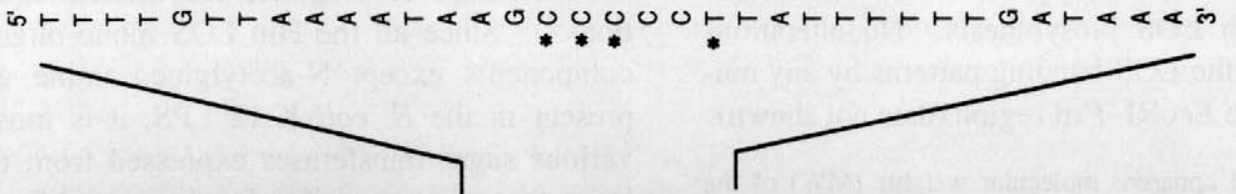

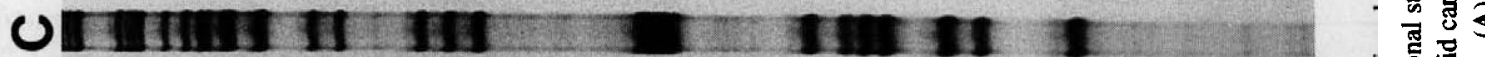

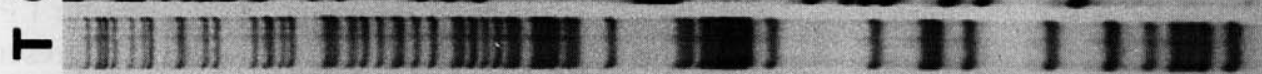

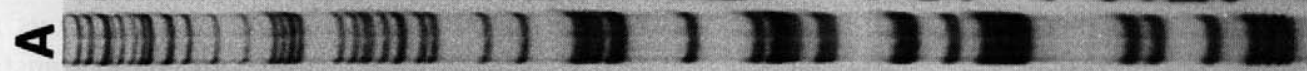

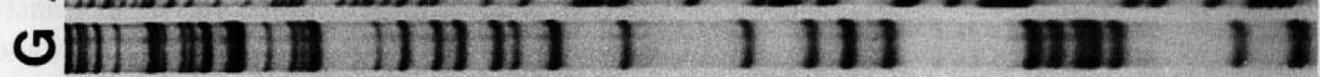

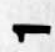


A

TAGCTTGTCTATGTTGATTTTGTTAAAATAAGCCCCTTATITTTTGATA

ORF1 $\rightarrow$

AACGAACGCTCGAATTCATCAAAAGTGCGGTGATATTTATGAAAGTTTTT

$M \quad R \quad F$

AAAGACAGTGTTATTTACCTTGTTGGGGAATTATCGTCAAAGTTAGTTCC

$\begin{array}{lllllllllllllllll}\mathrm{K} & \mathrm{D} & \mathrm{S} & \mathrm{V} & \mathrm{I} & \mathbf{Y} & \mathrm{L} & \mathrm{V} & \mathrm{G} & \mathbf{E} & \mathrm{L} & \mathbf{S} & \mathbf{S} & \mathrm{R} & \mathrm{L} & \mathrm{V} & \mathbf{P}\end{array}$

TTTTTTACTTTTGCCTTATTTATCCCGTAAATTGGGCGTAGAAGGGTATG

$\begin{array}{lllllllllllllllll}\text { F } & L & L & L & P & Y & L & S & R & K & \text { L } & \text { G } & \text { V } & \text { E } & \text { G } & \text { Y } & \text { G }\end{array}$ GCTCACTTTCT

S I $\mathbf{S}$

B TITTTCGCTECTTGATTAATCGAACCGCATTGTTGAATTTCTTTGAGTAA

$\begin{array}{lllllllllllllllll}\mathbf{N} & \mathbf{R} & \mathbf{A} & \mathbf{A} & \mathbf{Q} & \mathbf{N} & \mathbf{I} & \mathbf{S} & \mathbf{G} & \mathbf{C} & \mathbf{Q} & \mathbf{Q} & \mathbf{I} & \mathbf{E} & \mathbf{K} & \mathbf{L} & \mathbf{L}\end{array}$ ACGAACTCGTTITGGATCGATAAAAAGTGCTTGTTGAAGTTTAATTGTGA

$\begin{array}{llllllllllllllll}\mathbf{R} & \mathbf{V} & \mathbf{R} & \mathbf{K} & \mathbf{P} & \mathbf{D} & \mathbf{I} & \mathbf{F} & \mathbf{L} & \mathbf{A} & \mathbf{Q} & \mathbf{Q} & \mathbf{L} & \mathbf{K} & \mathbf{I} & \mathbf{T}\end{array}$

$\leftarrow$ ORF7

GTAAAATTTCGGTGTTTTTCATITTGCTGTCCCTAAGTAATCCTAAGTGG

$\begin{array}{lllllllll}\text { L } & \text { L } & \text { I } & \mathbf{E} & \mathrm{T} & \mathbf{N} & \mathrm{K} & \mathbf{M}\end{array}$

TAAGCCTAAGGCTTATATATTTTACTAAATAATTTTTCTTTTGCTATATG

ATTGTTATTTATTTCAATATATAATCAAGTAAATAACGTTTAATCGTAGG

ORF8 $\rightarrow$

AGATGATTCATGAAAAAATTAACTAAAATTTCAACCGCACTITTAATCGC

$\begin{array}{llllllllllllll}M & K & K & L & T & K & I & S & T & A & L & L & I & A\end{array}$

AGGATTAGGCTTTTCTTTTGCGGCATCCGCTAAAGTGACTGTATTTGCAG

$\begin{array}{lllllllllllllllll}G & L & G & F & S & F & A & A & S & A & K & V & T & V & F & A & A\end{array}$

CCGCTTCAATGACTGATGCTTTACAACAAGTAGCAAAAGATTATGCAAAA

$\begin{array}{lllllllllllllllllllllll}A & S & M & T & D & A & L & Q & Q & V & A & K & D & Y & A & K\end{array}$

Fig. 6 - Promoter regions of the ORFs I (A), ORF7 and ORF8 (B) of the lsg locus. The asterisks indicate the positions of the transcription start sites determined by primer extension analysis. The arrows indicate the directions of the ORFs. The -10 regions for the promoters are underlined and the -35 regions double-underlined. The sequences for the primers used for primer extension analysis are underlined by dots.

similar to those previously observed for transposon insertions elsewhere in the ORF5/ORF6 sequences. 10 LOS from mutants with insertions that were mapped to the ORF7 region had greater than 50\% increased binding of MAb 2F2, while MAb 6E4 binding was equivalent to that observed with the LOS from the parental strain (Fig. 7). Insertions mapped to ORF8 had no observable effect on LOS biosynthesis. No alterations were observed in the LOS banding patterns by any mutation in the $1.7 \mathrm{~kb}$ EcoRI-Pstl region (data not shown).

Table. Deduced and apparent molecular weights (MW) of the products of the ORFs within the $l s g$ locus

\begin{tabular}{ccc}
\hline ORF & $\begin{array}{c}\text { Deduced } \\
\text { MW (kDa) }\end{array}$ & $\begin{array}{c}\text { Apparent } \\
\text { MW (kDa) }\end{array}$ \\
\hline 1 & 46 & 40 \\
2 & 36 & 36 \\
3 & 26 & 25 \\
4 & 31 & $?$ \\
5 & 34 & 34 \\
6 & 31 & 30 \\
7 & 29 & 28 \\
8 & 22 & 23,22 \\
\hline
\end{tabular}

\section{DISCUSSION}

Previous studies suggested that the $7.4 \mathrm{~kb}$ Hib $l s g$ locus contains the sequences necessary for the 3 sequential modifications of the LPS of $E$. coli strain K12. ${ }^{5}$ Analysis of Hib mutants with transposon insertions within the $5.6 \mathrm{~kb}$ EcoRI fragment also indicated 3 modifications. ${ }^{10}$ Since all the Hib LOS mono-oligosaccharide components except $\mathrm{N}$-acetylglucosamine are already present in the $E$. coli K-12 LPS, it is most probably various sugar transferases expressed from the Hib lsg locus that are responsible for these modification of the existing $E$. coli LPS. In $E$. coli and $S$. typhimurium, all the $r a$ genes encoding sugar transferases for LPS core structure, with the exception of $r a E$, are present as a cluster. $^{27}$ Thus, it is likely that the lsg locus should contain a series of genes coding for sugar transferases. The sequence analysis of the $7.4 \mathrm{~kb}$ fragment and database search for the proteins homologous with the 8 ORFs, however, failed to show significantly high homology to any known sugar transferase, and it was not possible to deduce functions of the products of the ORFs based on the sequence homology. But it is of interest that the multiple sequence alignment show the presence of conserved motifs in the products of ORF5 
and ORF6, and the RfbB, RfbJ and RfbX proteins, suggesting that they are all related. The product of ORF1 does not contain the conserved motifs shown in Figure 3 , but shares homology with the RfbX protein (20\% identity, $50 \%$ similarity). These $r b$ genes of Enterobacteriaceae encode proteins involved in $\mathrm{O}$ antigen synthesis. ORF275 in the $E$. coli $r b$ gene cluster, whose product is highly homologous to the ORF6 protein, is thought to encode a galactosyltransferase. ${ }^{28} \mathrm{Al}$ though Hib LOS does not contain $\mathrm{O}$ antigen, ORF1, ORF5 and ORF6 may encode proteins with similar functions in LOS biosynthesis.

The products of ORF7 and ORF8 have homology with the proteins encoded by molA and molB which are suggested to be involved in molybdenum transport in $A$. vinelandii, ${ }^{26}$ but the exact functions of the MolA and MolB proteins have not been determined. We have evi- dence that ORF 7 may not code for any structural proteins but may code for a regulatory protein. First, although the EcoRI-PstI fragment containing ORF7 and ORF8 was unable to alter $E$. coli LPS, transposon insertions within ORF7 of Hib genome resulted in approximately 2 -fold increased binding of MAb $2 F 2$ without changing the binding of $6 \mathrm{E} 4$. These mutations did not alter the LOS band patterns on SDS-PAGE. Secondly, in vitro transcription/translation studies also showed that the deletion of ORF7 resulted in an increase in the expression of protein products from the other ORFs and that this is not due to decrease in competition for enzymes or substrates in the experimental system (data not shown).

DNA sequence analysis of the $7.4 \mathrm{~kb} / \mathrm{sg}$ locus suggested that ORFs $1-6$ are composed of 2 operons, each of which contains 3 ORFs (Fig. 1A) The transcription
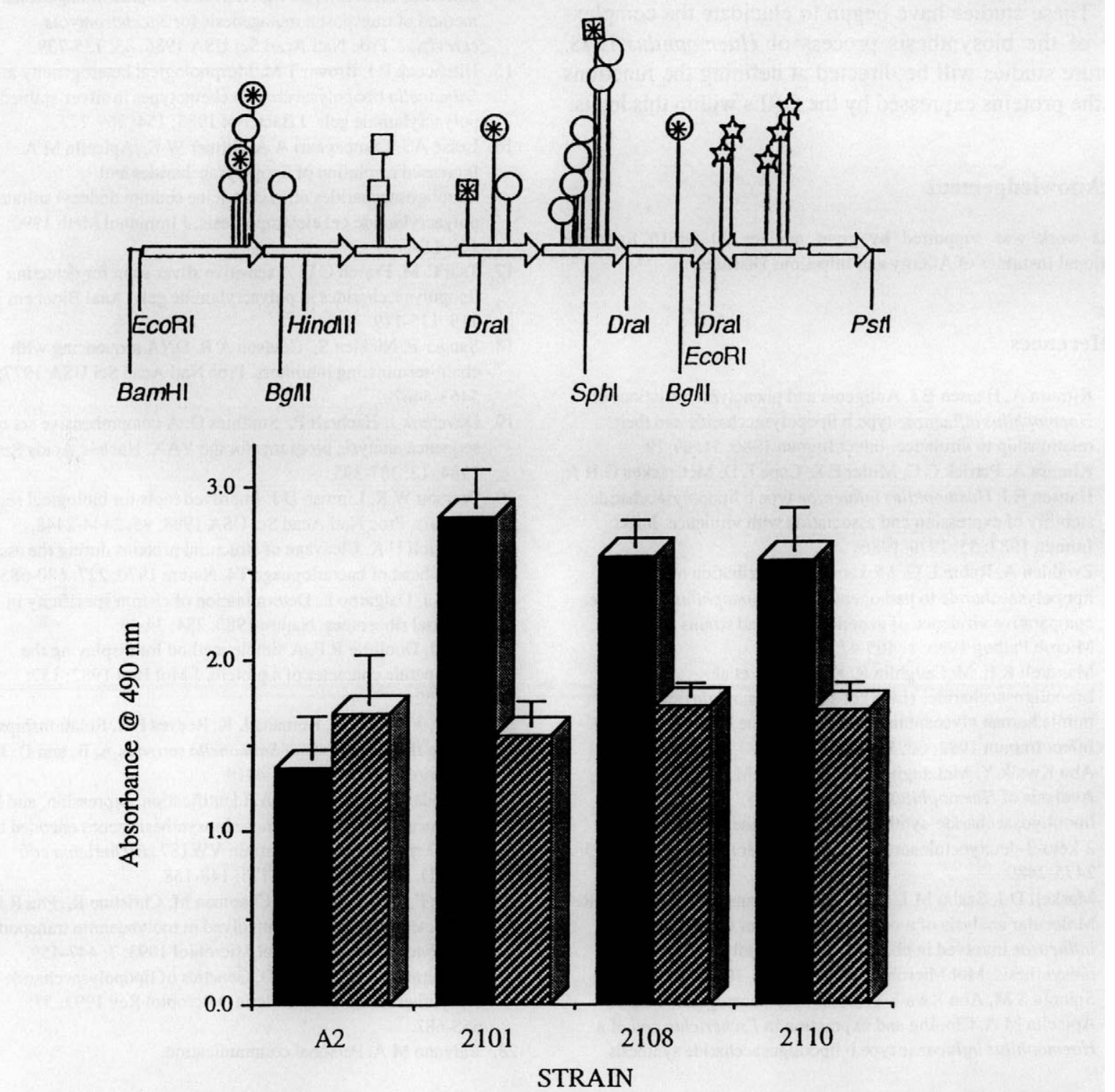

Fig. 7 - (A) Locations of $\mathrm{m}$ - $\mathrm{Tn} 3(\mathrm{Cm})$ insertion sites as determined by genomic Southern hybridization. The insertion sites are marked on the map with open reading frames. Symbols indicated the effect of transposon insertion on the binding of the LOS to monoclonal antibodies $6 \mathrm{E} 4$ and $2 \mathrm{~F} 2$. Symbols: circles $6 \mathrm{E} 4 / 2 \mathrm{~F} 2$ reduced binding; squares $6 \mathrm{E} 4 / 2 \mathrm{~F} 2$ negative phenotype; stars $2 \mathrm{~F} 2$ increased binding; asterisks multiple insertions mapped to these sites. (B) Reactivity of $H$. influenzae type b A2 and isogenic $l s g$ ORF 7 mutant strains 2101, 2108 and 2110 with monoclonal antibodies $6 \mathrm{E} 4$ and $2 \mathrm{~F} 2$. Each value represents the average of 11 replicate samples \pm SD from a single assay. Assays were performed at least twice giving similar results. Filled bar represents MAb 2F2 binding, hatched bar represents MAb 6E4 binding. 
start site for ORF1 was determined by primer extension analysis. The primer extension analysis for the putative second operon containing ORFs 4-6, using a few oligonucleotides complementary to the region of DNA just upstream or downstream of the putative ATG codon of ORF4, yielded several faint bands at very high positions on a gel. It was, therefore, not possible to determine the exact position of the primer extended products. At this point, it is not clear whether ORFs 46 are transcribed as a part of the first operon with ORFs 1-3 and these faint bands are from cleaved RNA products or whether transcription of the second operon starts at a very upstream region from the ORF3 coding region. ORF7 and ORF8 are transcribed in opposite directions, sharing a promoter region. This is an interesting feature, considering the possibility that these 2 ORFs encode regulatory proteins, whose expressions, in turn, could be regulated by other factors.

These studies have begun to elucidate the complexity of the biosynthesis process of Haemophilus LOS. Future studies will be directed at defining the functions of the proteins expressed by the ORFs within this locus.

\section{Acknowledgement}

This work was supported by grant number AI 24616 from the National Institutes of Allergy and Infectious Diseases.

\section{References}

1. Kimura A, Hansen E J. Antigenic and phenotypic variations of Haemophilus influenzae type b lipopolysaccharide and their relationship to virulence. Infect Immun 1986; 51: 69-79.

2. Kimura A, Patrick C C, Miller E E, Cope L D, McCracken G H Jr, Hansen $\mathrm{E}$ J. Haemophilus influenzae type b lipopolysaccharide: stability of expression and association with virulence. Infect Immun 1987; 55: 1979-1986.

3. Zwahlen A, Rubin L G, Moxon E R. Contribution of lipopolysaccharide to pathogenicity of Haemophilus influenzae: comparative virulence of genetically-related strains in rats. Microb Pathog 1986; 1: 465-473.

4. Mandrell R E, McLaughlin R, Kwaik Y A et al. Lipooligosaccharides (LOS) of some Haemophilus species mimic human glycosphingolipids, and some LOS are sialyated. Infect Immun 1992; 60: 1322-1328.

5. Abu Kwaik Y, McLaughlin R E, Apicella M A, Spinola S M. Analysis of Haemophilus influenzae type b lipooligosaccharide-synthesis genes that assemble or expose a 2-keto-3-deox yoctulosonic acid epitope. Mol Microbiol 1991; 5: 2475-2480.

6. Maskell D J, Szabo M J, Butler P D, Williams A E, Moxon E R. Molecular analysis of a complex locus from Haemophilus influenzae involved in phase-variable lipopolysaccharide biosynthesis. Mol Microbiol 1991; 5: 1013-1022.

7. Spinola S M, Abu Kwaik Y, Lesse A J, Campagnari A A Apicella M A. Cloning and expression in Escherichia coli of a Haemophilus influenzae type $b$ lipooligosaccharide synthesis gene(s) that encodes a 2-keto-3-deoxyoctulosonic acid epitope Infect Immun 1990; 58: 1558-1564

8. Weiser J N, Lindberg A A, Manning E J, Hansen E J, Moxon E R. Identification of a chromosomal locus for expression of lipopolysaccharide epitopes in Haemophilus influenzae. Infect Immun 1989; 57: 3045-3052.

9. Weiser J N, Love J M, Moxon E R. The molecular mechanism of phase variation of $H$. influenzae lipopolysaccharide. Cell 1989; 59: 657-665.

10. McLaughlin R, Spinola S M, Apicella M A. Generation of lipooligosaccharide mutants of Haemophilus influenzae type b. J Bacteriol 1992; 174: 6455-6459.

11. Phillips N J, Apicella M A, Griffiss J, Gibson B W. Structural studies of the lipooligosaccharides from Haemophilus influenzae type b strain A2. Biochem 1993; 32: 2003-2012.

12. Herriott R M, Meyer E M, Vogt $M$. Defined nongrowth media for stage II development of competence in Haemophilus influenzae. J Bacteriol 1970; 101: 517-524.

13. Sambrook J, Fritsch E F, Maniatis T. Molecular cloning: a laboratory manual, 2nd edn. NY: Cold Spring Harbor Laboratory Press, 1989.

14. Seifert S, Chen E Y, So M, Heffron F. Shuttle mutagenesis: A method of transposon mutagenesis for Saccharomyces cerevisiae. Proc Natl Acad Sci USA 1986; 83: 735-739.

15. Hitchcock P J, Brown T M. Morphological heterogeneity among Salmonella lipopolysaccharide chemotypes in silver-stained polyacrylamide gels. J Bacteriol 1983; 154: 269-277.

16. Lesse A J, Campagnari A A, Bittner W E, Apicella M A. Increased resolution of lipopolysaccharides and lipooligosaccharides utilizing tricine sodium dodecyl sulfate polyacrylamide gel electrophoresis. J Immunol Meth 1990; 126 : 109-117.

17. Tsai C-M, Frasch C E. A sensitive silver stain for detecting lipopolysaccharides in polyacrylamide gels. Anal Biochem 1982 119: 115-119.

18. Sanger F, Nicklen $S$, Coulson A R. DNA sequencing with chain-terminating inhibitors. Proc Natl Acad Sci USA 1977; 74 : 5463-5467.

19. Devereux J, Haeberli P, Smithies $O$. A comprehensive set of sequence analysis programs for the VAX. Nucleic Acids Res 1984; 12: 387-395.

20. Pearson W R, Lipman D J. Improved tools for biological sequence analysis. Proc Natl Acad Sci USA 1988; 95: 2444-2448.

21. Laemmli U K. Cleavage of structural proteins during the assembly of the head of bacteriophage T4. Nature 1970; 227: 680-685.

20. Shine J, Dalgarno L. Determination of cistron specificity in bacterial ribosomes. Nature 1980; $254: 34-38$.

23. Kyte J, Doolittle R F. A simple method for displaying the hydropathic character of a protein. J Mol Biol 1982; 157: 105-132.

24. Liu D, Verma N K, Romana L K, Reeves P R. Relationships among the ifb regions of Salmonella serovars A, B, and D. J Bacteriol 1991; 173: 4814-4819.

25. Marolda C L, Valvano $M A$. Identification, expression, and DNA sequence of the GDP-mannose biosynthesis genes encoded by the 07 rfb gene cluster of strain VW187 (Escherichia coli 07:K1). J Bacteriol 1993; 175: 148-158.

26. Luque F, Mitchenall L A, Chapman M, Christine R, Pau R N. Characterization of genes involved in molybdenum transport in Azotobacter vinelandii. Mol Microbiol 1993; 7: 447-459.

27. Schnaitman C A, Klena J D. Genetics of lipopolysaccharide biosynthesis in enteric bacteria. Microbiol Rev 1993; 57 : 655-682.

28. Valvano M A. Personal communication. 